\title{
METHODOLOGY FOR TRANSITION PROBABILITIES DETERMINATION IN A MARKOV DECISION PROCESSES MODEL FOR QUALITY-ACCURACY MANAGEMENT
}

\author{
UDC: $005.336 .1: 519.217 .6 / .8$ \\ Original Scientific Paper \\ Katerina MITKOVSKA-TRENDOVA ${ }^{1}$, Robert MINOVSKI ${ }^{2}$ and Dime BOSHKOVSKI ${ }^{1}$ \\ ${ }^{1}$ Goce Delcev University in Stip, Military Academy Gen. Mihailo Apostolski in Skopje, 1000 Skopje, Vasko \\ Karangeleski bb, Republic of Macedonia \\ E-mail: katerina.trendova@morm.gov.mk \\ ${ }^{2}$ Ss. Cyril and Methodius University in Skopje, Faculty of Mechanical Engineering, Department of Industrial \\ Engineering and Management, 1000 Skopje, Karpos II bb, Republic of Macedonia.
}

Paper received: 16.10.2014.; Paper accepted: 18.11.2014.

\begin{abstract}
The main goal of the presented research is to define a methodology for determination of the transition probabilities in a Markov Decision Process on the example of optimization of the qualityaccuracy through optimization of its main measure (percent of scrap) in a Performance Measurement System. This research had two main driving forces. First, today's urge for introduction of more robust, mathematically founded methods/tools in different enterprise areas, including PMSs. Second, since Markov Decision Processes are chosen as such tool, certain shortcomings of this approach had to be handled. Exactly the calculation of the transition probabilities is one of the weak points of the Markov Decision Processes. The proposed methodology for calculation of the transition probabilities is based on utilization of recorded historical data and they are calculated for each possible transition from a state after one run to a state after the following run of the influential factor (e.g. machine). The methodology encompasses several steps that include: collecting different data connected to the percent of scrap and their processing according to the needs of the methodology, determination of the limits of the states for every influential factor, classification of the data from real batches according to the determined states and calculation of the transition probabilities from one state to another state for every action. However, the implementation of the Markov Decision Process model with the proposed methodology for calculation of the transition probabilities resulted in optimal policy that showed significant differences in the percent of scrap, compared to the real situation when the optimization of the percent of scrap was done heuristically $(5.2107 \%$ versus $13.5928 \%)$.
\end{abstract}

Keywords: Markov decision processes, transition probabilities, performance measurement systems, quality-accuracy, percent of scrap.

\section{INTRODUCTION}

Today's turbulent and dynamic market environment forces the enterprises to seek for more and more comprehensive and complex tools for managing their overall activities and processes. The need for more accurate and fast decisions is bigger than ever. In that direction, almost every aspect of enterprises' functioning is experiencing dramatic changes. Performance Measurement Systems (PMSs) are one of the significant tools for coping with those new circumstances. They are already accepted as a foundation of several management approaches/philosophies, like Total Quality Management (TQM), Lean Management, Strategic Management, etc. Their main intention is to picture and quantify the present state of the enterprise, pinpointing in that way the directions for further improvement of the enterprise. In order to accomplish this, they usually divide the overall enterprise functioning in several areas (called key elements of success, key performance elements, etc.). For the needs of the quantification, this division should go to such portions of enterprise functioning that can be measured with one single measure for the whole enterprise. In that sense in 
COMPASS (COmpany's Management Purpose ASSistance) (Minovski, 1999), the PMS that is used in this research, 18 so called subkey elements of success (subKEs) are defined. Some of them are time-reliability, quality-accuracy, and qualitycapability. This can be expected from one overall PMS that wants to encompass the whole enterprise. The problems begin when it comes to generation of the actions for further improvement of the situation (in COMAPSS called Success Factors - SF). The number of influential factors on such state is quite big. Correspondingly (since one factor can be facilitated with several actions - SF) the number of $\mathrm{SF}$ is even bigger. In such situations, the generation of the SF is done mainly heuristically. This was the main reason to start the research that will attain more robust tools for decision making in this point. Since every subKE is a different story with different knowledge background, demanding different models, the initial research focused on the one subKE - quality-accuracy, measured through the percent of scrap. The main reasons were its importance for the enterprises and the wideness of its implementation.

In the research, Markov Decision Processes (MDPs) are used for accomplishment of the aforementioned goal. Their analysis showed that they can be excellent choice due to their proved opportunities and advantages. They are seen as one of the fundamental models, simple and easy to understand, where control of the evolution of a dynamic system can be obtained and has an insight of the condition of the system in every stage of the process. The success of the application can be attributed to the existence of efficient algorithms for finding optimal solutions for MDP models (Puterman, 1994). Still they have certain disadvantages. The curse of dimensionality (resulting in limited number of analyzed factors in situations when we want to obtain exact optimal policy) and the calculation of the transition probabilities are seen as the most crucial.

In that way, the main research challenge of this paper is to define a methodology for determination of the transition probabilities in a MDP on the example of optimization of the quality-accuracy through optimization of its main measure (percent of scrap) in one PMS.

So, the frames of this research can be presented in several circles, Figure 1.

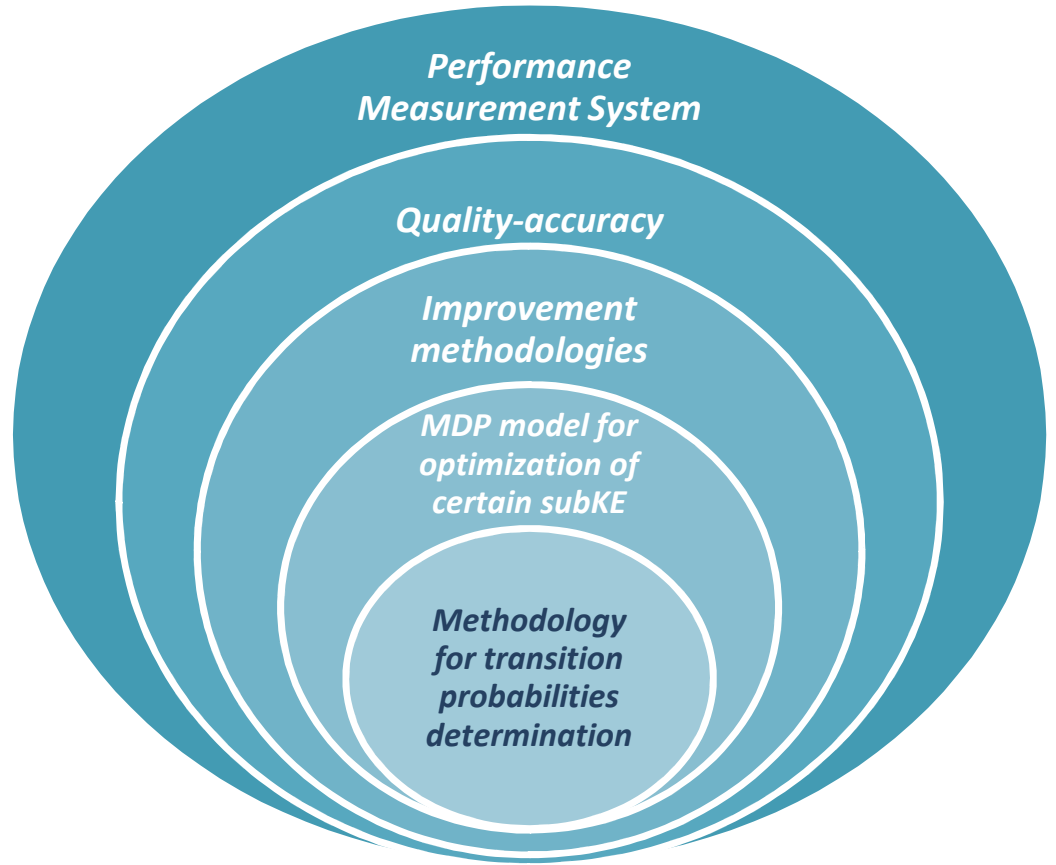

Figure 1: The frames of the research

It has to be stressed that the PMS and qualityaccuracy are just used to explain the methodology through practical example. The implementation of the proposed methodology goes beyond them and can be implemented in every occasion when MDPs are implemented and needed historical data can be obtained.

\section{MATHEMATICAL MODEL OF FOUR- DIMENSIONAL MDP FOR OPTIMIZATION OF CERTAIN SUBKE}

All stages of the research are summarized in a model for optimal decision policy and success factors generation for the critical element quality- 
accuracy of the selected PMS, in order to implement it in a real enterprise. The steps of this methodology are: 1) defining the real problem 2) scanning of the enterprise and data collection 3) selection of most influential factors 4) creation of the mathematical model 5) design and selection of software 6) analysis of real data and their adaptation to the needs of the model 7) software implementation and output processing 8) analysis of the results 9) unification of results for all jobs and comparison to real condition of the enterprise 10) making report with conclusions and recommendations to the enterprise. Figure 2 shows the flowchart of the research process.

The stage 4 (Creation of the mathematical model) from the above methodology is the hearth of the whole process. In the following text, the main features of this stage will be elaborated.

The condition of a system - one job place is modeled in order to follow and optimize the percent of scrap. SubKE quality-accuracy is influenced by many factors. Some of them are the machine, the operator, the tools, the materials, the environment, the method, the shifts, etc. In order to avoid too complex mathematical model, but on the other hand to get sufficiently realistic model that will show the condition of the system, only the most influential factors or causes of scrap are chosen: the machine, the operator, the tools and the materials. The model should provide following of the induced scrap divided by influential factors and overall. Stochastic processes described by random variables are defined, which represent the conditions of the machine, the operator, the tools and the materials regarding percent of scrap caused by these causes. A stochastic process represented by vector random process is created, consisted of the four above mentioned random processes that reflect the individual and the total scrap production for this system. The conditions of these four factors regarding the percent of scrap they caused are recorded and classified in finite number of states at the end of each run of the work place, which give the values of the four random/stochastic processes. The recorded real data help to find the transition probabilities of each possible transition from a state after one run to a state after the following run of the work place, under the influence of the corrective actions, for each of the influential factors. The transition probabilities are unaffected by the states of the prior work place runs. Because of that, it is concluded that the stochastic processes are Markov chains and it is assumed that they are discrete-time, finite and homogenous Markov chains. For each of the Markov chains finite action spaces are defined, considering the available decision alternatives i.e. corrective actions. Each transition effected by each action is followed by certain revenue. All these elements define the one-dimensional MDPs. The created four-dimensional vector random process is also MDP. Its structure is defined in terms of the one-dimensional MDPs structures.

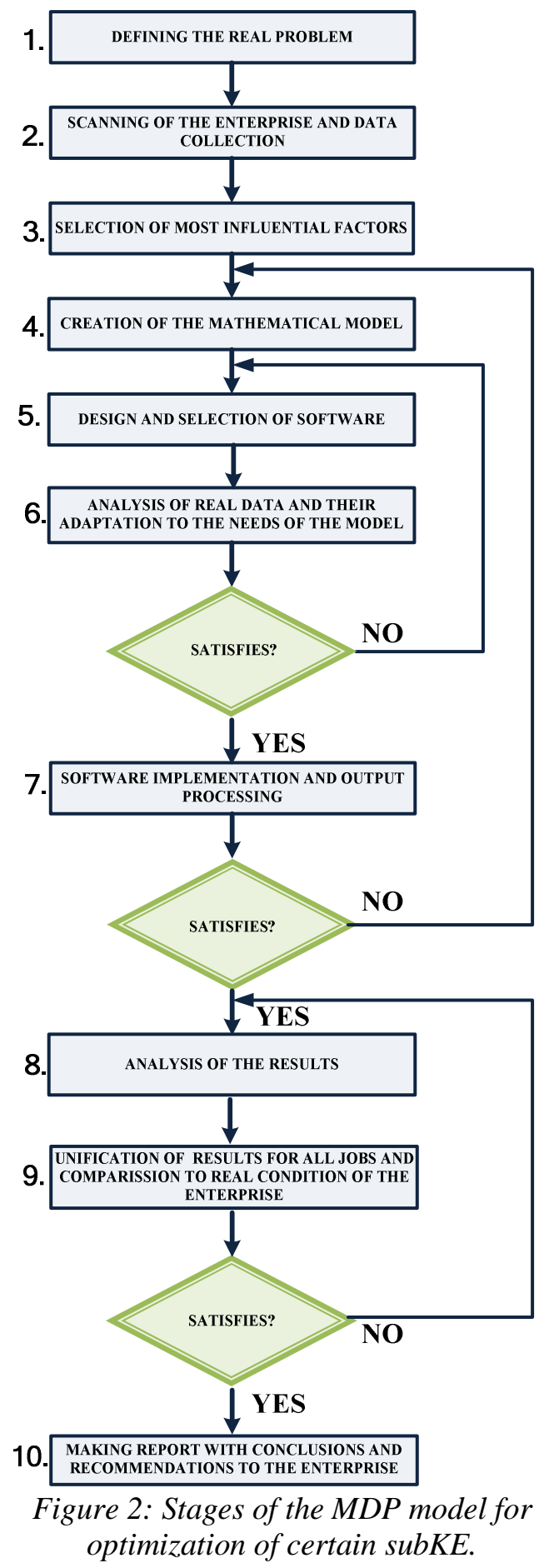

The first MDP is the random process "the condition of the machine after every run of the work place". The random variable $X_{n}^{1}$ is the 
condition of the machine in a discrete moment $n$, which represents the stochastic process $\left\{X_{n}^{1} \mid n \in \mathbb{N}\right\}$ i.e. the first Markov chain. At any point of time $n$, the condition of the machine can be classified in one of several possible states and the random variable $X_{n}^{1}$ in a given moment takes values from the defined state space for the condition of the machine. In every discrete moment of time the random variable $X_{n}^{1}$ takes values from the same state space, and further this random process is denoted only by $X_{1}$ for every transition moment and this is also counts for the other stochastic processes. All random processes, i.e. the given Markov chains are mutually independent. The random process $X_{2}$ is the second Markov chain, which is "the condition of the operator after every run of the work place", expressed by the caused percent of scrap from the operator. The third Markov chain is the random process $X_{3}$, which is "the condition of the machine tools after every run of the work place", expressed by the caused percent of scrap from the tools. The fourth Markov chain is the random process $X_{4}$, which is "the condition of the materials after each run of the work place", expressed by the caused percent of scrap from the materials. The random process $X_{l}, l \in\{1,2,3,4\}$, takes $\left|R_{X_{l}}\right|=n_{l}$ values. The sets of values for these stochastic processes are $R_{X_{l}}=\left\{x_{1}^{l}, x_{2}^{l}, x_{3}^{l}\right\}, n_{l}=3$. The same notations for the states of the one-dimensional MDPs and the values of associated random processes are used in order to simplify the notations. $A_{l}, l \in\{1,2,3,4\}$ denote the sets of primary actions (decisions, alternatives) for the one-dimensional MDPs and $\left|A_{l}\right|=m_{l}$. Let $A_{l}=\left\{a_{1}^{l}, a_{2}^{l}, a_{3}^{l}\right\},\left|A_{l}\right|=m_{l}=3$.

\section{DETERMINATION OF TRANSITION PROBABILITIES}

One of the main challenges one has to face when dealing with MDP model is the difficulty in determining or estimating the transition probabilities. The accuracy of the estimates of the transition probabilities determines the accuracy of the Markov model. These estimates are frequently made relying on inadequate or incorrect data. Sometimes they are only based on expert opinion. It is better if they are derived from cohort studies, but again may be imprecise or subject to selection bias. Small samples or short following of the modeled process produce confidence intervals that are large relative to the transition probabilities (Black et al., 1997). In the area of maintenance, the determination of transition probabilities usually is done using one of two methods (Ortiz-García et al.,
2006). The usual approach is to observe, from historical data, the way in which a system transits from a state to another, for every stage of the process, and use this to derive/estimate the transition probabilities. The other way, is to use experienced engineers to estimate the probabilities using expert opinion. But many other ideas and methods are developed in different fields of Markov processes applications such as in (Bath and Lal, 1990; Black et al., 1997; Dent, 1973; Jones, 2005; Jung, 2006; Kaur and Rajarshi, 2012; Mitkovska-Trendova et al., 2008; Ortiz-García et al., 2006; Puz et al., 2010; Salkin et al., 1976; Theil and Rey, 1966; Welton and Ades, 2005). One of them is approximation with some probability distribution characteristic for the process.

This paper proposes and describes the development of methodology for transition probabilities estimation in the frames of the mathematical model that advances quality-accuracy management. Historical data are used to determine the primary transition probabilities. Very often, these data are unavailable, or difficult to get, and in the meantime are subject to various influences and changes in the environment. That also implies changes of their values. The model assumes that transition probabilities do not change over time, i.e. Markov chains are homogenous. The transition probabilities are denoted with $p_{i j}^{k}(l)$, and they are the conditional probabilities that the random process $X_{l}$ takes value $x_{j}^{l}$ if its previous value was $x_{i}^{l}$, under the effect of the action $a_{k}^{l}, i \in\{1,2,3\}$, $j \in\{1,2,3\}, k \in\{1,2,3\}, l \in\{1,2,3,4\}$.

The state space $\mathcal{S}$ for the four-dimensional MDP is defined as the set of all ordered quadruplets formed by the elements of the value sets of $X_{1}, X_{2}, X_{3}$ and $X_{4}, \quad$ and that is $\mathcal{S}=\left\{\left(x_{i}^{1}, x_{j}^{2}, x_{k}^{3}, x_{l}^{4}\right), i \in\right.$ $\left\{1,2, \ldots,\left|R_{X_{1}}\right|\right\}, j \in\left\{1,2, \ldots,\left|R_{X_{2}}\right|\right\}, k \in$ $\left.\left\{1,2, \ldots,\left|R_{X_{3}}\right|\right\}, l \in\left\{1,2, \ldots,\left|R_{X_{4}}\right|\right\}\right\}$, and it consists of $\quad|\mathcal{S}|=\left|R_{X_{1}}\right| \cdot\left|R_{X_{2}}\right| \cdot\left|R_{X_{3}}\right| \cdot\left|R_{X_{4}}\right|=n_{1} \cdot n_{2}$. $n_{3} \cdot n_{4}$ states. The number of all possible transitions between the states of the system is calculated by $|\mathcal{S}|^{2}$. The action space is defined similarly as the state space $\mathcal{A}=\left\{\left(a_{i}^{1}, a_{j}^{2}, a_{k}^{3}, a_{l}^{4}\right), i \in\left\{1,2, \ldots,\left|A_{1}\right|\right\}, j \in\right.$ $\left\{1,2, \ldots,\left|A_{2}\right|\right\}, k \in\left\{1,2, \ldots,\left|A_{3}\right|\right\}, l \in$ $\left.\left\{1,2, \ldots,\left|A_{4}\right|\right\}\right\}$ and it consists of $|\mathcal{A}|=\left|A_{1}\right| \cdot\left|A_{2}\right|$. $\left|A_{3}\right| \cdot\left|A_{4}\right|=m_{1} \cdot m_{2} \cdot m_{3} \cdot m_{4}$ actions. Using the fact that the random variables are independent, a method for calculating the joint transition probabilities is proposed, knowing those transition 
probabilities in the one-dimensional MDPs. The number of transition probabilities is $|\mathcal{S}|^{2} \cdot|\mathcal{A}|$. For the

$\left(x_{i_{1}}^{1}, x_{i_{2}}^{2}, x_{i_{3}}^{3}, x_{i_{4}}^{4}\right)$

$\stackrel{\left(a_{k_{1}}^{1}, a_{k_{2}}^{2}, a_{k_{3}}^{3}, a_{k_{4}}^{4}\right)}{\longrightarrow}\left(x_{j_{1}}^{1}, x_{j_{2}}^{2}, x_{j_{3}}^{3}, x_{j_{4}}^{4}\right)$, where $i_{1}, j_{1} \in$ $\left\{1,2, \ldots,\left|R_{X_{1}}\right|\right\}, \quad i_{2}, j_{2} \in\left\{1,2, \ldots,\left|R_{X_{2}}\right|\right\}, \quad i_{3}, j_{3} \in$ $\left\{1,2, \ldots,\left|R_{X_{3}}\right|\right\}, \quad i_{4}, j_{4} \in\left\{1,2, \ldots,\left|R_{X_{4}}\right|\right\}, \quad k_{1} \in$ $\left\{1,2, \ldots,\left|A_{1}\right|\right\}, \quad k_{2} \in\left\{1,2, \ldots,\left|A_{2}\right|\right\}, \quad k_{3} \in$ $\left\{1,2, \ldots,\left|A_{3}\right|\right\}, \quad k_{4} \in\left\{1,2, \ldots,\left|A_{4}\right|\right\}$, the transition probability is calculated by $p_{i_{1} j_{1}}^{k_{1}}(1) \cdot p_{i_{2} j_{2}}^{k_{2}}(2)$. $p_{i_{3} j_{3}}^{k_{3}}(3) \cdot p_{i_{4} j_{4}}^{k_{4}}(4)$.

The primary transition matrices are stochastic and that implies that the created transition matrices for the four-dimensional MDP are also stochastic. If $n_{1}=n_{2}=n_{3}=n_{4}=3, \quad m_{1}=m_{2}=m_{3}=$ $m_{4}=3$, the system has 12 primary transition matrices $3 \times 3$, and that is $9 \cdot 12=108$ primary transition probabilities. The state space has $3^{4}=81$ states, and the number of actions in the action space is the same. The number of all possible transitions between the states of the system is $3^{8}=6561$, and $|\mathcal{S}|^{2} \cdot|\mathcal{A}|=3^{8} \cdot 3^{4}=$ $3^{12}=531441$ is the number of the joint transition probabilities. The calculated values of the primary transition probabilities and the primary revenues are input for the software specially designed to calculate the joint transition probabilities and the associated revenues for the four-dimensional MDP. The relatively small number of states and actions in the example allows to use exact optimization technique and because of the relatively short time of finding the optimal solution, the discounted policy iteration method to solve the MDP is chosen for the research.

\section{REAL APPLICATION OF THE MODEL AND RESULTS FOR THE TRANSITION PROBABILITIES}

The methodology for optimal decision policy and success factors generation including the mathematical model was implemented in an enterprise from the graphic industry in Macedonia. It was used to help the improvement of the decision-making in quality-accuracy management, in order to minimize the scrap and the associated costs. Although data are collected for several machines for different functions of the production process, only the results for the job place printing machine that enterprise uses the most and is dominant regarding the scrap production are presented. Also, only the results of obtaining the transition probabilities are given.

The design studio and the printing machine are connected with proper software. The machine also has software to record total scrap production, but not divided by causes, so these records makes the operator manually in special daily reports, in order to follow causes for scrap. Later these records are processed in special forms. The collected data for scrap production available for the research referred to about 400 successive orders (printing machine runs). These data included number of scrap for each batch, possible causes for scrap, appropriate corrective actions and associate costs. These information helped the modeling of the real problem. Then the collected data were adapted and processed in forms, specially designed for the needs of the research. The approved/planned amount of make-ready sheets in this enterprise meets the available information about the amount of make-ready sheets in other enterprises from the graphic industry. In their forms only the produced scrap after the setup is considered. Considering the expert opinion it is concluded that it is more appropriate to use the measure percent of scrap and the average of percents of scrap in defining the states of MDPs. According to the opinion of the experts from the enterprise and the collected real data for the successive batches with different number of sheets printed on the monitored machine, it is decided to consider three intervals of percents of scrap for every scrap cause. The limits are determined according to the average of percents of scrap for all batches, for each of the causes. The collected data of available corrective actions were used to define action spaces for the MDPs and three types of actions are defined for each cause. Real data give information of all transitions from one state to another under the influence of certain action. It is assumed that some of the transitions are unfeasible and the associated transition probabilities are zero. These assumptions are that under the influence of action $a_{1}^{l}$ the condition of the factor can't "improve", and under the influence of action $a_{2}^{l}$ and action $a_{3}^{l}$ the condition of the factor can't deteriorate. Thus some of the transition probabilities are determined, and the transition matrices are: 


\begin{tabular}{|c|c|c|c|}
\hline$a_{1}^{l}$ & $x_{1}^{l}$ & $x_{2}^{l}$ & $x_{3}^{l}$ \\
\hline$x_{1}^{l}$ & $p_{11}^{1}(l)$ & $p_{12}^{1}(l)$ & $p_{13}^{1}(l)$ \\
\hline$x_{2}^{l}$ & 0 & $p_{22}^{1}(l)$ & $p_{23}^{1}(l)$ \\
\hline$x_{3}^{l}$ & 0 & 0 & 1 \\
\hline
\end{tabular}

\begin{tabular}{|c|c|c|c|}
\hline$a_{2}^{l}$ & $x_{1}^{l}$ & $x_{2}^{l}$ & $x_{3}^{l}$ \\
\hline$x_{1}^{l}$ & 1 & 0 & 0 \\
\hline$x_{2}^{l}$ & $p_{21}^{2}(l)$ & $p_{22}^{2}(l)$ & 0 \\
\hline$x_{3}^{l}$ & $p_{31}^{2}(l)$ & $p_{32}^{2}(l)$ & $p_{33}^{2}(l)$ \\
\hline
\end{tabular}

\begin{tabular}{|c|c|c|c|}
\hline$a_{3}^{l}$ & $x_{1}^{l}$ & $x_{2}^{l}$ & $x_{3}^{l}$ \\
\hline$x_{1}^{l}$ & 1 & 0 & 0 \\
\hline$x_{2}^{l}$ & $p_{21}^{3}(l)$ & $p_{22}^{3}(l)$ & 0 \\
\hline$x_{3}^{l}$ & $p_{31}^{3}(l)$ & $p_{32}^{3}(l)$ & $p_{33}^{3}(l)$ \\
\hline
\end{tabular}

Figure 3 shows the collected historical data for the percents of scrap caused by the machine for the monitored sample. Table 1 summarizes the defined states and actions for each of the four MDPs.

Historical data for the percent of scrap caused by the machine

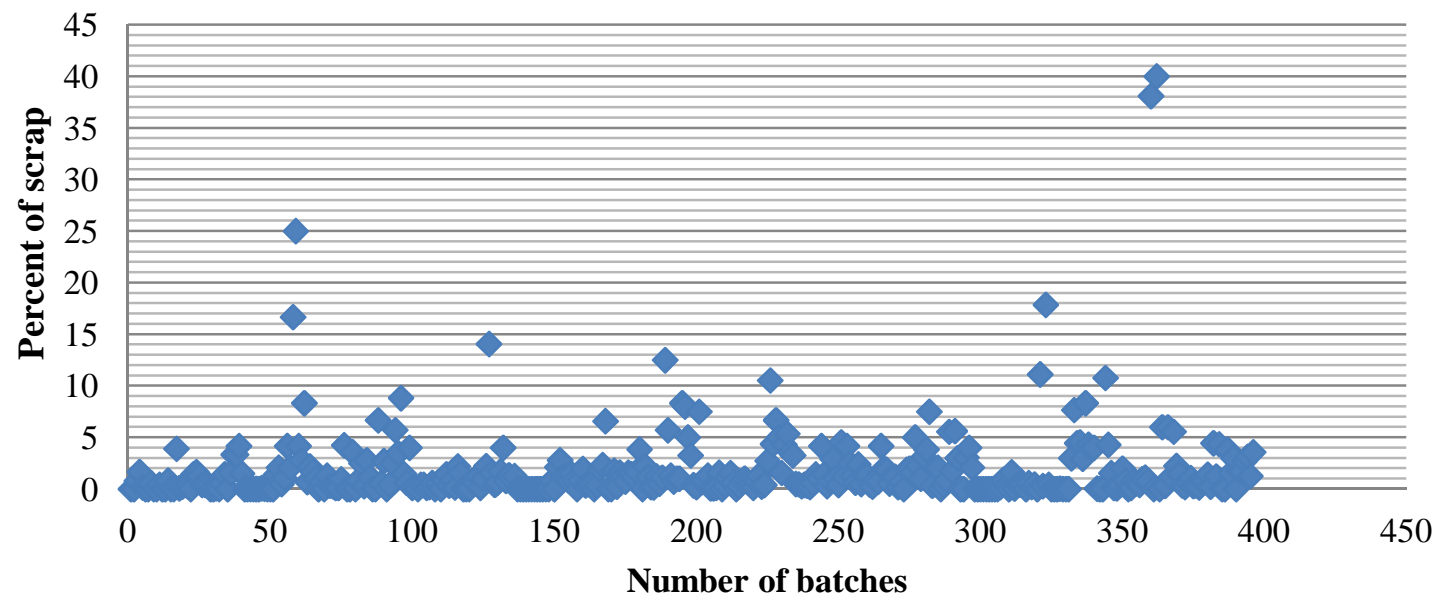

Figure 3: Historical data for the percent of scrap caused by the machine.

Table 1: States and actions for the four causes/influencing factors.

\begin{tabular}{|l|l|l|l|l|}
\hline & \multicolumn{1}{|c|}{ Machine } & \multicolumn{1}{c|}{ Operator } & \multicolumn{1}{c|}{ Tools } & \multicolumn{1}{c|}{ Materials } \\
\hline State 1 & From 0\% to 1.8\% & From 0\% to 8.25\% & From 0\% to 2.4\% & From 0\% to 1.1\% \\
\hline State 2 & Over 1.8\% to 3.6\% & Over 8.25\% to 16.5\% & Over 2.4\% to 4.8\% & Over 1.1\% to 2.2\% \\
\hline State 3 & Over 3.6\% & Over 16.5\% & Over 4.8\% & Over 2.2\% \\
\hline Action 1 & Do nothing & Do nothing & Do nothing & Do nothing \\
\hline Action 2 & $\begin{array}{l}\text { Cleaning, } \\
\text { maintenance, } \\
\text { servicing } \\
\text { performed by } \\
\text { operators employed } \\
\text { and trained in the } \\
\text { company }\end{array}$ & $\begin{array}{l}\text { Pause, replacement, } \\
\text { warning }\end{array}$ & $\begin{array}{l}\text { Cleaning, gluing, } \\
\text { setting, etc. }\end{array}$ & $\begin{array}{l}\text { Adapting, adjusting, } \\
\text { cleaning, addition, } \\
\text { dilution, etc. }\end{array}$ \\
\hline Action 3 & $\begin{array}{l}\text { Servicing performed } \\
\text { by personnel from } \\
\text { companies specialized } \\
\text { and authorized for } \\
\text { these machines }\end{array}$ & $\begin{array}{l}\text { Consultation, } \\
\text { explanation, training } \\
\text { by more experienced } \\
\text { and more trained } \\
\text { employee }\end{array}$ & $\begin{array}{l}\text { Replacement with } \\
\text { new tools }\end{array}$ & $\begin{array}{l}\text { Replacement of } \\
\text { bad materials }\end{array}$ \\
\hline
\end{tabular}

After the classification of the batches in one of the three defined states for each of the four influential factor i.e. each Markov chain, all transitions are counted and classified according to cause, influential action, and the starting and the next 
state. The transition probabilities are calculated using the processed data with the formula

$$
p_{i j}^{k}(l)=\frac{N_{i j}^{k}(l)}{N_{i}^{k}(l)}
$$

where $N_{i}^{k}(l)$ denotes the number of times the onedimensional MDP described by the random variable $l$ was in state with index $i$, under the influence of primary action with index $k$, and
$N_{i j}^{k}(l)$ denotes the number of times it made transition from state with index $i$ to state with index $j$, under the influence of primary action with index $k$. The notations of the primary states and actions are simplified identifying them with their indexes. For the given example, $i \in\{1,2,3\}$, $j \in\{1,2,3\}, k \in\{1,2,3\}, \quad l \in\{1,2,3,4\}$. Thus the calculated transition probabilities can be represented in 12 transition probabilities matrices, or summarized in Table 2:

Table 2: Transition probabilities for the one-dimensional MDPs.

\begin{tabular}{|c|c|c|c|c|c|c|c|c|c|c|}
\hline \multirow{2}{*}{$\boldsymbol{p}_{\boldsymbol{i}}^{\boldsymbol{k}}(\boldsymbol{l})$} & $\boldsymbol{i}$ & $\mathbf{1}$ & $\mathbf{1}$ & $\mathbf{1}$ & $\mathbf{2}$ & $\mathbf{2}$ & $\mathbf{2}$ & $\mathbf{3}$ & $\mathbf{3}$ & $\mathbf{3}$ \\
\cline { 2 - 12 } & $\boldsymbol{j}$ & $\mathbf{1}$ & $\mathbf{2}$ & $\mathbf{3}$ & $\mathbf{1}$ & $\mathbf{2}$ & $\mathbf{3}$ & $\mathbf{1}$ & $\mathbf{2}$ & $\mathbf{3}$ \\
\hline \multirow{4}{*}{$\boldsymbol{l}=\mathbf{1}$} & $\boldsymbol{k}=\mathbf{1}$ & 0.811 & 0.0893 & 0.0997 & 0 & 0.2941 & 0.7059 & 0 & 0 & 1 \\
\cline { 2 - 12 } & $\boldsymbol{k}=\mathbf{2}$ & 1 & 0 & 0 & 0.92 & 0.08 & 0 & 0.5106 & 0.2341 & 0.2553 \\
\cline { 2 - 12 } & $\boldsymbol{k}=\mathbf{3}$ & 1 & 0 & 0 & 0.6667 & 0.3333 & 0 & 0.625 & 0.125 & 0.25 \\
\hline \multirow{3}{*}{$\boldsymbol{l}=\mathbf{2}$} & $\boldsymbol{k}=\mathbf{1}$ & 0.7556 & 0.1370 & 0.1074 & 0 & 0.4737 & 0.5263 & 0 & 0 & 1 \\
\cline { 2 - 11 } & $\boldsymbol{k}=\mathbf{2}$ & 1 & 0 & 0 & 0.875 & 0.125 & 0 & 0.3889 & 0.1667 & 0.4444 \\
\cline { 2 - 12 } & $\boldsymbol{k}=\mathbf{3}$ & 1 & 0 & 0 & 0.9565 & 0.0435 & 0 & 0.4889 & 0.1555 & 0.3556 \\
\hline \multirow{3}{*}{$\boldsymbol{l}=\mathbf{3}$} & $\boldsymbol{k}=\mathbf{1}$ & 0.7658 & 0.1264 & 0.1078 & 0 & 0.48 & 0.52 & 0 & 0 & 1 \\
\cline { 2 - 12 } & $\boldsymbol{k}=\mathbf{2}$ & 1 & 0 & 0 & 0.9655 & 0.0345 & 0 & 0.4082 & 0.1632 & 0.4286 \\
\cline { 2 - 11 } & $\boldsymbol{k}=\mathbf{3}$ & 1 & 0 & 0 & 0.75 & 0.25 & 0 & 0.6875 & 0.1875 & 0.125 \\
\hline \multirow{3}{*}{$\boldsymbol{l}=\mathbf{4}$} & $\boldsymbol{k}=\mathbf{1}$ & 0.8022 & 0.1079 & 0.0899 & 0 & 0.4211 & 0.5789 & 0 & 0 & 1 \\
\cline { 2 - 11 } & $\boldsymbol{k}=\mathbf{2}$ & 1 & 0 & 0 & 0.8 & 0.2 & 0 & 0.4063 & 0.2187 & 0.3750 \\
\cline { 2 - 11 } & $\boldsymbol{k}=\mathbf{3}$ & 1 & 0 & 0 & 0.8571 & 0.1429 & 0 & 0.45 & 0.35 & 0.2 \\
\hline
\end{tabular}

All determined values for the transition probabilities are input for specially designed software for calculation of the transition probabilities and associate revenues for the fourdimensional MDP. For the considered dimensions of the model the output from the designed software are 81 transition probabilities matrices with dimensions $81 \times 81$ and 81 revenue matrices with the same dimensions. They are very big and it is not possible to show them in the paper. They also are input for the policy iteration method.

For certain discount factor, and the obtained optimal policy, the expected percent of scrap for the optimal policy per transition step can be calculated. This means that under the optimal policy, for each subsequent batch/lot from now on, one can expect an average percent of scrap that reflects the improvement of the condition of the system. The concluding comparison is that the average value of percent of scrap for the selected sample of the real system was $13.5928 \%$, and with the obtained optimal decision policy proposed by the mathematical model it will decrease to $5.2107 \%$. The I/P matrixes are used to present the situation in the enterprise before and after the improvement (Minovski and Jovanoski, 2000). The improved value for the performance of the sub-key element quality-accuracy is entered in the revised I/P (Importance/Performance) matrix (the initial matrix was designed for the situation before the improvement). It is clear that the subKE qualityaccuracy was in the problematic quadrant with high importance and low performance. After the improvement, this subKE moved to the quadrant with high importance and high performance. Figure 4 shows the revised I/P matrix.

\section{RESULTS AND DISCUSSION}

The implementation of the MDP model with the proposed methodology for calculation of the transition probabilities resulted in optimal policy that showed significant improvement in the area of quality-accuracy. The difference in the percent of scrap (as the main measure for the qualityaccuracy) of the new situation compared to the real situation (when the optimization of the percent of scrap was done heuristically) is quite big. These figures are $5.2107 \%$ versus $13.5928 \%$ accordingly. 


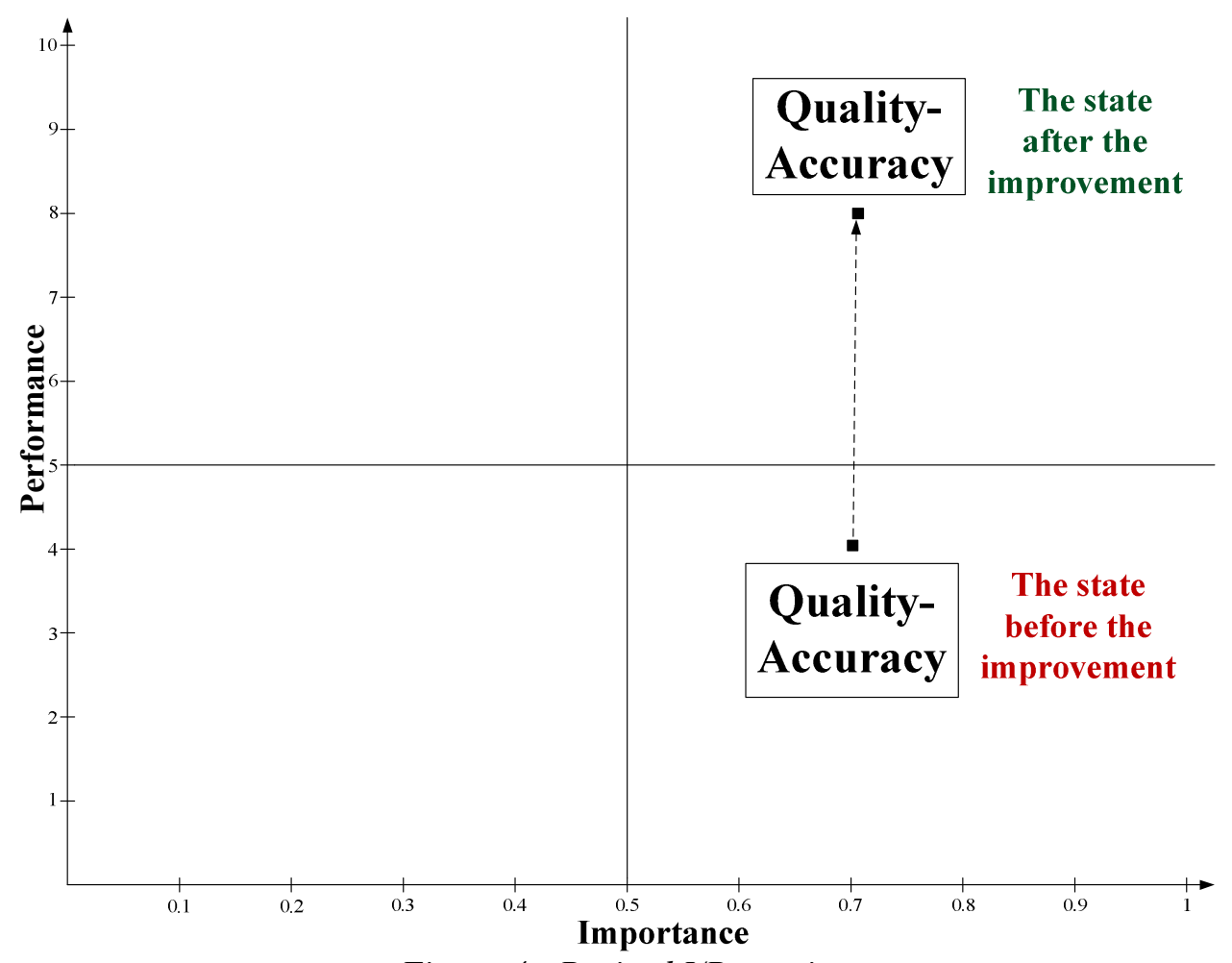

Figure 4:. Revised I/P matrix.

As mentioned before, the importance of the accurate calculation of the transition probabilities is one of the crucial factors for implementation of the MDPs. Namely, the accuracy of the transition probabilities determines the accuracy of the MDP itself. In that direction, this paper proposes implementation of the following summarized methodology for their determination.

- Gathering data for every factor (it is assumed that the factors are already determined in the principal methodology).

- These data encompass the percent of scrap for batches in certain period of time, including the causes (influential factors) of scrap and undertaken corrective actions.

- Adaptation of the data

- In case the data are obtained in other format (number of scrap pieces), the percent of scrap should be calculated. This percent of scrap is calculated separately for every influential factor for every batch.

- Also, the corrective actions are classified in several groups of actions according to their similarity (in this case, three actions for every influential factor are defined).

- Calculation of the average percents of scrap for the whole sample per influential factor

- Determination of the number of states for every influential factor and calculation of the control limits for every state. If there are $l$ influential factors and the number of states for every influential factor is $n_{l}$, the possible number of transitions for every influential factor is $n_{l}^{2}$. In case of 3 states for every influential factor, the possible number of transitions for every influential factor is 9. For every action there are 9 such transitions and the total number of transitions for every factor is 27 .

- For every batch the classification of every influential factor in previously determined states is done

- Counting of the number of transitions from one state to another consequent state under the influence of every action is performed

- Calculation of the transition probabilities (according to the formulas given in the previous text)

\section{CONCLUSIONS}

The calculated probabilities are just the estimation of the real probabilities in frames of some confident intervals. Theoretically, the calculation of such intervals demands gathering the data in longer period of time and utilization of certain statistical analysis (Ortiz-García et al., 2006). Still, even if this kind of analysis is done, environmental changes might question the obtained results. Anyhow, this is one of the main directions for further research in this area. 
The methodology is based on the thorough collection of the historical data which will obtain the percentage of scrap for every influential factor and every corrective action for every consecutive batch in a certain period of time. This kind of monitoring of percent of scrap is not always available in the praxis and this may be a certain obstacle in the implementation of the proposed methodology.

However, the implementation of the MDP model with the proposed methodology for calculation of the transition probabilities resulted in optimal policy that showed significant differences in the percent of scrap between the new (optimized) and the initial situation.

\section{REFERENCES}

Bhat, U. N., \& Lal, R. (1990). Attribute Control Charts for Markov Dependent Production Processes. IIE Transactions, 22(2), 181-188.

Black, W. C., Nease, R. F., \& G., W. H. (1997). Determining Transition Probabilities from Mortality Rates and Autopsy Findings. Medical Decision Making, 17(1), 67-93.

Dent, W. (1973). The Estimation of Nonstationary Transition Probabilities. Management Science, 20(3), 308-312.

Jones, M.T. (2005). Estimating Markov Transition Matrices Using Proportions Data: An Application to Credit Risk, IMF Working Paper.

Jung, J. (2006). Estimating Markov Transition Probabilities between Health States in the HRS Dataset (pp. 1-42). Indiana University: Mimeo.

Kaur, I., \& Rajarshi, M. B. (2012). Ridge Regression for Estimation of Transition Probabilities from Aggregate Data. Communications in Statistics -
Simulation and Computation, 41(4), 524-530.

Minovski, R. (1999). Creation of the Model for Overall Restructuring of the Enterprises. $\mathrm{PhD}$ thesis, Faculty of Mechanical Engineering, Skopje, Macedonia.

Minovski, R., \& Jovanoski, D. (2000). Utilisation of the Importance/Performance (I/P) Matrixes in a Model for Enterprise Restructuring. Proceedings of the 44th European Quality Congress (Vol. 1, pp. 79-86). Budapest.

Mitkovska-Trendova, K., Minovski, R., \& Jovanoski, D. (2008). Finite-Stage Markov Decision Processes in Inventory Management. Proceedings of the International Conference for Entrepreneurship, Innovation and Regional Development - ICEIRD (pp. 631-640). Ohrid, Macedonia.

Ortiz-García, J. J., Costello, S. B., \& Snaith, M. S. (2006). Derivation of Transition Probability Matrices for Pavement Deterioration Modeling. Journal of Transportation Engineering, 132(2), 141161.

Puterman, M. (1994). Markov Decision Processes: Discrete Stochastic Dynamic Programming (1st ed.). New York, NY, USA: John Wiley \& Sons Inc.

Puz, G., Radic, J., \& Stipanovic-Olsakovic, I. (2010). Novi model za stohasticku analizu trajnosti mostova. GRAĐEVINAR, 62(4), 287-297.

Salkin, M. S., Just, R. E., \& Cleveland, O. A., Jr. (1976). Estimation of Nonstationary Transition Probabilities for Agricultural Firm Size Projection. The Annals of Regional Science, 10(1), 71-82.

Theil, H., \& Rey, G. (1966). A Quadratic Programming Approach to the Estimation of Transition Probabilities. Management Science, 12(9), 714-721.

Welton, N. J., \& Ades, A. E. (2005). Estimation of Markov Chain Transition Probabilities and Rates from Fully and Partially Observed Data: Uncertainty Propagation, Evidence Synthesis, and Model Calibration. Medical Decision Making, 25(6), 633645. doi: 10.1177/0272989x05282637 